\title{
XII. On the stability of two rectilinear vortices of compressible fluid moving in an incompressible liquid
}

\section{Bibhutibhusan Datta M.Sc.}

To cite this article: Bibhutibhusan Datta M.Sc. (1920) XII. On the stability of two rectilinear vortices of compressible fluid moving in an incompressible liquid, Philosophical Magazine Series 6, 40:235, 138-148, DOI: 10.1080/14786440708636104

To link to this article: http://dx.doi.org/10.1080/14786440708636104

曲 Published online: 08 Apr 2009.

Submit your article to this journal $₫$

Џll Article views: 2

Q View related articles $\sqsubset$ 
XII. On the Stability of two Rectilinear Vortices of Compressible Fluid moving in an Incompressible Liquid. By Bibhutibhusan DatTa, M.Sc., Lecturer in Applied Mathematics, University of Calcutta, India**

THE stability of the circular form in two rectilinear 1 vortices has been discussed by Sir J. J. Thomson $\dagger$ for incompressible fluid.

Dr. Chree $\ddagger$ attempted to extend his treatment to a compressible fluid but did not succeed except in some special cases. The object of the present paper is to complete the work begun by Dr. Chree.

When the vortex-lines are straight lines parallel to the axis of $z$, the velocity components $u, v$ at any point $(x, y)$ in the fluid are given by $\S$

$$
u=-\frac{\partial \phi}{\partial x}-\frac{\partial \psi}{\partial y}, \quad v=-\frac{\partial \phi}{\partial y}+\frac{\partial \psi}{\partial x} ; \quad .
$$

hence

$$
\nabla_{1}^{2} \phi=-\theta, \quad \nabla_{1}^{2} \psi=\zeta, \quad \text {. . . }
$$

where

$$
\theta=\frac{d u}{d x}+\frac{d v}{d y}, \quad \nabla_{1}^{2}=\frac{\partial^{2}}{\partial x^{2}}+\frac{\partial^{2}}{\partial y^{2}} \text {. }
$$

Then by the theory of attraction,

$$
\left.\begin{array}{l}
\phi=-\frac{1}{2 \pi} \iint \theta^{\prime} \log r d x^{\prime} d y^{\prime}+\phi_{0}, \\
\psi=\frac{1}{2 \pi} \iint \zeta^{\prime} \log r^{\prime} d x^{\prime} d y^{\prime}+\psi_{u,}
\end{array}\right\}
$$

$\theta^{\prime}, \zeta^{\prime}$ being respectively the value of $\theta$ and $\zeta$ at the point $\left(x^{\prime}, y^{\prime}\right)$ and

$$
y^{2}=\left\{\left(x-x^{\prime}\right)^{2}+\left(y-y^{\prime}\right)^{2}\right\}^{\frac{1}{2}}, \quad \nabla_{1}^{2} \phi_{0}=0, \quad \nabla_{1}^{2} \psi_{0}=0 .
$$

If $\frac{d}{d t}$ denote partial differentiation, and $\frac{\mathrm{D}}{\mathrm{D} t}$ differentiation

* Communicated by the Author.

† 'Motion of Vortex Rings,' p. 71.

† Messenger of Mathematics, vol. x vii. p. 113 (1888).

$\S$ Lamb, 'Hydrodynamics,' 4th ed. p. 213 (1916). 
Stability of Rectilinear Vortices of Compressible Fluid. 139 following the fluid, the equation of continuity is

$$
\begin{gathered}
\frac{d \rho}{d t}+\frac{d \rho u}{d x}+\frac{d \rho v}{d y}=0, \\
\frac{1}{\rho} \frac{\mathrm{D} \rho}{\mathrm{D} t}+\theta=0 . \\
\text { or } \quad \phi=\frac{1}{2 \pi} \iint \frac{1}{\rho^{\prime}} \frac{\mathrm{D} \rho^{\prime}}{\mathrm{D} t} \log r d x^{\prime} d y^{\prime}+\phi_{0} . . .
\end{gathered}
$$

If $\rho_{0}$ be the mean value of the density of the fluid at the cross section $\sigma$ of a circular vortex, defined by the relation

$$
\iint \rho^{\prime} d x^{\prime} d y^{\prime}=\rho_{0} \sigma, \quad \text {. . . . . . }
$$

we get

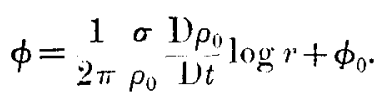

Again, the equation of continuity is

$$
\begin{gathered}
\frac{\mathrm{D} \cdot \rho_{0} \sigma}{\mathrm{D} t}=0 \\
\text { or } \quad \frac{1}{\rho_{0}} \frac{\mathrm{D} \rho_{0}}{\mathrm{D} t}+\frac{1}{\sigma} \frac{\mathrm{D} \sigma}{\mathrm{D} t}=0 . \\
\phi=-\frac{1}{2 \pi} \frac{\mathrm{D} \sigma}{\mathrm{D} t} \log r+\phi_{0} . \quad . \quad . \quad .
\end{gathered}
$$

Hence

Since $\sigma$ always refers to a definite column of fluid, there is no difference between $\frac{\mathrm{D} \sigma}{\mathrm{D} t}$ and $\frac{d \sigma}{d t}$.

Let us consider a truly circular cross section of radius $a$; if the vorticity be the same at any instant at all points of the section, we have, when $r>a$,

$$
\left.\begin{array}{l}
\phi=-\frac{1}{2 \pi} \frac{d \sigma}{d t} \log r+\mathrm{C}, \\
\psi=\frac{\kappa}{2 \pi} \log r+\mathrm{D} ;
\end{array}\right\} .
$$

where $\kappa$ is the strength of the vortex, $C$ and $D$ being 
$140 \mathrm{Mr}$. B. Datta on Stability of two Rectilinear Vortices of constants. Also when $r<a$,

$$
\left.\begin{array}{l}
\phi=-\frac{1}{4 \pi a^{2}} \frac{d \sigma}{d t} r^{2}+\mathrm{C}^{\prime}, \\
\psi=\frac{\kappa}{4 \pi a^{2}} r^{2}+\mathrm{D}^{\prime} .
\end{array}\right\}
$$

Let the equation of the boundary of the cross section at any instant be given by

$$
r=a+\Sigma\left(\alpha_{n} \cos n \theta+\beta_{n} \sin n \theta\right), \quad . \quad .
$$

where $\alpha_{n}$ and $\beta_{n}$ are functions of $t$, independent of $\theta$, and very small compared with $a$.

Outside the vortex, $r>a$, let

$$
\left.\begin{array}{l}
\phi=\mathrm{C}-\frac{1}{2 \pi} \frac{d \sigma}{d t} \log r+\Sigma\left(\mathrm{E}_{n} \cos n \theta+\mathrm{F}_{n} \sin n \theta\right)\left(\frac{a}{r}\right)^{n}, \\
\psi=\mathrm{D}+\frac{\kappa}{2 \pi} \log r+\Sigma\left(\mathrm{A}_{n} \cos n \theta+\mathrm{B}_{n} \sin n \theta\right)\left(\frac{a}{r}\right)^{n} ;
\end{array}\right\}
$$

inside the vortex, $r<a$,

$$
\left.\begin{array}{l}
\phi^{\prime}=\mathrm{C}^{\prime}-\frac{1}{4 \pi a^{2}} \frac{d \sigma}{d t} r^{2}+\Sigma\left(\mathrm{E}_{n}{ }^{\prime} \cos n \theta+\mathrm{F}_{n}{ }^{\prime} \sin n \theta\right)\left(\frac{r}{a}\right)^{n}, \\
\psi^{\prime}=\mathrm{D}^{\prime}+\frac{\kappa}{4 \pi a^{2}} r^{2}+\Sigma\left(\mathrm{A}_{n}{ }^{\prime} \cos n \theta+\mathrm{B}_{n}{ }^{\prime} \sin n \theta\right)\left(\frac{r}{a}\right)^{n} .
\end{array}\right\} .
$$

Since $\phi$ and $\psi$ must be continuous when crossing the boundary, we at once get, neglecting products of small quantities,

$$
\mathrm{A}_{n}{ }^{\prime}=\mathrm{A}_{n}, \quad \mathrm{~B}_{n}{ }^{\prime}=\mathrm{B}_{n}, \quad \mathrm{E}_{n}{ }^{\prime}=\mathrm{E}_{n}, \quad \mathrm{~F}_{n}{ }^{\prime}=\mathrm{F}_{n} .
$$

The radial and tangential velocities for points just inside and just outside the vortex must be the same. Therefore when

$$
\begin{aligned}
& r=a+\Sigma\left(\alpha_{n} \cos n \theta+\beta_{n} \sin n \theta\right) \\
& \frac{1}{r} \frac{\partial \psi}{\partial \theta}+\frac{\partial \phi}{\partial r}=\frac{1}{r} \frac{\partial \psi^{\prime}}{\partial \theta}+\frac{\partial \phi^{\prime}}{\partial r} \\
& \text { and } \quad-\frac{\partial \psi}{\partial r}+\frac{\partial \phi}{r \partial \theta}=-\frac{\partial \psi^{\prime}}{\partial r}+\frac{\partial \phi^{\prime}}{r \partial \theta} .
\end{aligned}
$$


Compressible Fluid moving in Lurompressible Liquid.

Since products of small terms are neglected, these conditions become

$$
\frac{\partial \psi}{\partial r}=\frac{\partial \psi^{\prime}}{\partial r^{\prime}}, \frac{\partial \phi}{\partial r}=\frac{\partial \phi^{\prime}}{\partial r^{\prime}}
$$

Substituting the values of $\psi, \psi^{\prime}, \phi, \phi^{\prime}$ from (10), (11) and equating coefficients of $\cos n \theta \operatorname{and} \sin n \theta$, we get

$$
\begin{aligned}
& \mathrm{A}_{n}=-\frac{\kappa}{2 \pi n a} \alpha_{n}, \quad \mathrm{~B}_{n}=-\frac{\kappa}{2 \pi n a} \beta_{n}, \\
& \mathrm{E}_{n}=\frac{1}{2 \pi n a} \frac{d \sigma}{d t} \alpha_{n}, \quad \mathrm{~F}_{n}=\frac{1}{2 \pi n a} \frac{d \sigma}{d t} \beta_{n} . .
\end{aligned}
$$

Hence outside the vortex, we have

$$
\left.\begin{array}{l}
\phi=\mathrm{C}-\frac{1}{2 \pi} \frac{d \sigma}{d t} \log r+\Sigma \frac{\frac{d \sigma}{d t}}{2 \pi n a}\left(\alpha_{n} \cos n \theta+\beta_{n} \sin n \theta\right)\left(\frac{a}{r}\right)^{n}, \\
\psi=\mathrm{D}+\frac{\kappa}{2 \pi} \log r-\Sigma \frac{\kappa}{2 \pi n a}\left(\alpha_{n} \cos n \theta+\beta_{n} \sin n \theta\right)\left(\frac{a}{r}\right)^{n} \cdot
\end{array}\right\}
$$

Suppose there are two vortices of strengths $\kappa, \kappa^{\prime}$; let their cross sections at time $t$ be $\sigma$ and $\sigma^{\prime}$ respectively, the distance between their centres being $c$. I et the radii of their cross sections be given by

$$
\left.\begin{array}{l}
\mathrm{R}=a+\Sigma\left(\alpha_{n}^{\prime} \cos n \theta+\beta_{n} \sin n \theta\right), \\
\mathrm{R}^{\prime}=b+\Sigma\left(\alpha_{n}^{\prime} \cos n \theta^{\prime}+\beta_{n}{ }^{\prime} \sin n \theta^{\prime}\right) .
\end{array}\right\}
$$

Let $\phi, \psi$ be the functions at an external point for the first and $\phi^{\prime}, \psi^{\prime}$ for the second. If $\left(r^{\prime}, \theta\right)$ and $\left(r^{\prime}, \theta^{\prime}\right)$ be the coordinates of a point referred to the centres of the two vortices, $\phi$ and $\psi$ will be given by (14), and

$$
\left.\begin{array}{l}
\phi^{\prime}=\mathrm{C}^{\prime}-\frac{1}{2 \pi} \frac{d \sigma^{\prime}}{d t} \log r^{\prime}+\Sigma \frac{\frac{d \sigma^{\prime}}{d t}}{2 \pi n b}\left(\alpha_{n}{ }^{\prime} \cos n \theta^{\prime}+\beta_{n}{ }^{\prime} \sin n \theta^{\prime}\right)\left(\frac{b^{\prime}}{r^{\prime}}\right)^{n}, \\
\psi^{\prime}=\mathrm{D}^{\prime}+\frac{\kappa^{\prime}}{2 \pi} \log r^{\prime}-\Sigma \frac{\kappa^{\prime}}{2 \pi n b}\left(\alpha_{n}{ }^{\prime} \cos n \theta^{\prime}+\beta_{n}{ }^{\prime} \sin n \theta^{\prime}\right)\left(\frac{b}{r^{\prime}}\right)^{n} .
\end{array}\right\}
$$

We shall fix our attention on the second vortex. If $\mathfrak{A}$ be the radial velocity of a point on it and $b \Theta$ the velocity perpendicular to the rudius vector, both relative to the centre 
142 Mr. B. Datta on Stafility of tivo Rectilinear Vortices of of the vortex, we have from (15)

$$
\begin{aligned}
\mathfrak{l i}=\frac{d l}{d t} & +\Sigma\left(\frac{d \alpha_{n}{ }^{\prime}}{d t} \cos n \theta^{\prime}+{ }^{d \beta_{n}{ }^{\prime}} d t \sin n \theta^{\prime}\right) \\
& -\Sigma n \Theta\left(\alpha_{n}{ }^{\prime} \sin n \theta^{\prime}-\beta_{n}{ }^{\prime} \cos n \theta^{\prime}\right) .
\end{aligned}
$$

Since products and powers of $\alpha_{n}{ }^{\prime}, \beta_{n}{ }^{\prime}$ are to be neglected, in (17) we may put

$$
\Theta=\frac{\kappa^{\prime}}{2 \pi b^{2}} \cdot \text {. . . . . . . }
$$

Ag:in

$$
\begin{aligned}
\mathbf{H}=-\frac{\partial \psi^{\prime}}{r^{\prime} \partial \theta^{\prime}}-\frac{\partial \phi^{\prime}}{\partial r^{\prime}} & -\frac{\partial \psi}{r^{\prime} \partial \theta^{\prime}}-\frac{\partial \phi}{\partial r^{\prime}}-\frac{\kappa}{2 \pi c} \sin \left(\theta^{\prime}-\epsilon\right) \\
& -\frac{1}{2 \pi c} \frac{d \sigma}{d t} \cos \left(\theta^{\prime}-\epsilon\right), \quad . \quad . \quad .
\end{aligned}
$$

where $r^{\prime}$ must be put equal to $b+\sum\left(\alpha_{n}{ }^{\prime} \cos n \theta^{\prime}+\beta_{n}{ }^{\prime} \sin n \theta^{\prime}\right)$ after differentiation, and $\epsilon$ is the angle made by the line joining the centres of the two vortices with its initial position. If $b / c$ be sinall, $\phi$ and $\psi$ can be expressed in terms of $r^{\prime}, \theta^{\prime}$, and $\epsilon$, such as

$$
\begin{aligned}
& \phi=\mathrm{C}-\frac{1}{2 \pi} \frac{d \sigma}{d t}\left[\log c+\sum_{s=1}^{\infty}\left(-\frac{1)^{s-1}}{s}\left(\frac{r^{\prime}}{c}\right)^{s} \cos s\left(\theta^{\prime}-\epsilon\right)\right]\right. \\
& +\Sigma \frac{1}{2 \pi n} \frac{d \sigma}{d t} \frac{a^{n-1}}{e^{n}}\left[\left(\alpha_{n} \cos n \epsilon+\beta_{n} \sin n \epsilon\right) \sum_{s=0}^{\infty}(-1)^{s}\right. \\
& \times \frac{n(n+1) \ldots(n+s-1)}{s !}\left(\frac{v^{\prime}}{\epsilon}\right)^{s} \cos s\left(\theta^{\prime}-\epsilon\right) \\
& +\left(\beta_{n} \cos n \epsilon-\alpha_{n} \sin n \epsilon\right) \sum_{s=1}^{\infty}(-1)^{s-1} \\
& \left.x^{n(n+1) \ldots(n+s-1)}\left(\frac{r^{\prime}}{c}\right)^{s} \sin s\left(\theta^{\prime}-\epsilon\right)\right], \\
& \psi=\mathrm{D}+\underset{2 \pi}{\kappa}\left[\log c+\sum_{s=1}^{\infty} \frac{(-1)^{s-1}}{s}\left(\frac{r^{\prime}}{c}\right)^{s} \cos s\left(\theta^{\prime}-\varepsilon\right)\right] \\
& -\Sigma_{2 \pi n} \frac{\kappa}{a^{n-1}} \cdot{ }_{c^{n}}^{a^{n}}\left[\left(\alpha_{n} \cos n \epsilon+\beta_{n} \sin n \epsilon\right) \sum_{s=0}^{\infty}(-1)^{s}\right. \\
& \times \frac{n(n+1) \ldots(n+s-1)}{s !}\left(\begin{array}{c}
r^{\prime} \\
c
\end{array}\right)^{s} \cos s\left(\theta^{\prime}-\epsilon\right) \\
& +\left(\beta_{n} \cos n \epsilon-\alpha_{n} \sin n \epsilon\right) \sum_{s=1}^{\infty}(-1)^{s-1} \\
& \left.\times \frac{n(n+1) \ldots(n+s-1)}{s !}\left(\frac{r^{\prime}}{c}\right)^{s} \sin s\left(\theta^{\prime}-\epsilon\right)\right] .
\end{aligned}
$$


Compressitle Fluid moving in Incompressible Liquid. 143 Substituting in (19) we get

$$
\begin{aligned}
& \mathbf{a}=-\Sigma \frac{\kappa^{\prime}}{2 \pi b^{2}}\left(\alpha_{n}{ }^{\prime} \sin u \theta^{\prime}-\beta_{n}{ }^{\prime} \cos n \theta^{\prime}\right)+\frac{1 d \sigma^{\prime}}{2 \pi b d t} \\
& +\frac{\kappa}{2 \pi} \sum_{s=1}^{\infty}(-1)^{s-1} \sin s\left(\theta^{\prime}-\epsilon\right) \frac{l_{s-1}^{s-1}}{c^{s}} \\
& \times\left[1+\frac{s-1}{b} \Sigma\left(\alpha_{n}{ }^{\prime} \cos n \theta^{\prime}+\beta_{n}{ }^{\prime} \sin n \theta^{\prime}\right)\right] \\
& +\sum \frac{\kappa}{2 \pi n} \frac{a^{n-1}}{e^{n}}\left[\left(\alpha_{n} \cos n \epsilon+\beta_{n} \sin n e\right) \sum_{s=1}^{\infty}(-1)^{s-1}\right. \\
& \times \frac{n(n+1) \ldots(n+s-1)}{(s-1) !} \frac{b^{s-1}}{c^{s}} \sin s\left(\theta^{\prime}-\epsilon\right) \\
& +\left(\beta_{n} \cos n \epsilon-\alpha_{n} \sin n \epsilon\right) \sum_{s=1}^{\infty}(-1)^{s-1} \\
& \left.\times \frac{n(n+1) \ldots(n+s-1)}{(s-1) !} \frac{b^{s-1}}{c^{s}} \cos s\left(\theta^{\prime}-\epsilon\right)\right] \\
& +\frac{1}{2 \pi} \frac{d \sigma}{d t} \sum_{s=1}^{\infty}(-1)^{s-1} \cos s\left(\theta^{\prime}-\epsilon\right) \frac{l^{s-1}}{c^{s}} \\
& \times\left[1+\frac{s-1}{b} \Sigma\left(\alpha_{n}{ }^{\prime} \cos n \theta^{\prime}+\beta_{n}{ }^{\prime} \sin n \theta^{\prime}\right)\right] \\
& -\sum \frac{1}{2 \pi n} \frac{d \sigma}{d t} \frac{a^{n-1}}{e^{n}}\left[\left(x_{n} \cos n \epsilon+\beta_{n} \sin n \epsilon\right) \sum_{s=1}^{\infty}(-1)^{s}\right. \\
& \times \frac{n(n+1) \ldots(n+s-1)}{(s-1) !} \frac{b^{s-1}}{r^{s}} \cos s\left(\theta^{\prime}-\epsilon\right) \\
& +\left(\beta_{n} \cos n \epsilon-\alpha_{n} \sin n \epsilon\right) \sum_{s=1}^{\infty}(-1)^{s-1} \\
& \left.\times \frac{n(n+1) \ldots(n+s-1)}{(s-1) !} \frac{b^{s-1}}{c^{s}} \sin s\left(\theta^{\prime}-\epsilon\right)\right] \\
& -\frac{\kappa}{2 \pi c} \sin \left(\theta^{\prime}-\epsilon\right)-\frac{1}{2 \pi c} d \sigma \cos \left(\theta^{\prime}-\epsilon\right) \text {. }
\end{aligned}
$$

The two values of $2 \mathrm{~h}$ as given by (17) and (20) must be the same; so that we can equate the terms independent of $\theta^{\prime}$ in either expression as well as the coefficients of the sines and cosines of multiples of $\theta^{\prime}$. The terms independent of $\theta^{\prime}$ simply verify the known result that $\frac{d \sigma^{\prime}}{d t}=2 \pi b \frac{d b}{d t}$. Equating the coefficients of $\cos \theta^{\prime}$, we have $\frac{d \alpha_{1}^{\prime}}{d t}=\frac{\kappa}{2 \pi c^{2}}\left(\beta_{1} \cos 2 \epsilon-\alpha_{1} \sin 2 \epsilon\right)+\frac{1}{2 \pi c^{2}} \frac{d \sigma}{d t}\left(\alpha_{1} \cos 2 \epsilon+\beta_{1} \sin 2 \epsilon\right) ;$ 


\section{$144 \mathrm{Mr}$. B. Datta on Stability of two Rectilinear Vortices of} so that to our degree of approximation $\frac{d a_{1}^{\prime}}{d t}=0$; similarly we get $\frac{d \beta_{1}{ }^{\prime}}{d t}=0$. Hence, so far as these terms are concerned, compressibility of fluid makes no change in the motion or shape of the vortices.

Equating the coefficients of $\cos 2 \theta^{\prime}$ and $\sin 2 \theta^{\prime}$, we get to the same degree of approximation

$$
\begin{aligned}
& \frac{d \alpha_{2}{ }^{\prime}}{d t}+\frac{\kappa^{\prime}}{2 \pi b^{2}} \beta_{2}{ }^{\prime}=\frac{\kappa b}{2 \pi c^{2}} \sin 2 \epsilon-\frac{b}{2 \pi c^{2}} \frac{d \sigma}{d t} \cos 2 \epsilon, . \\
& \frac{d \beta_{2}{ }^{\prime}}{d t}-\frac{\kappa^{\prime}}{2 \pi b^{2}} \alpha_{2}{ }^{\prime}=-\frac{\kappa b}{2 \pi \theta^{2}} \cos 2 \varepsilon-\frac{b}{2 \pi c^{2}} \frac{d \sigma}{d t} \sin 2 \epsilon .
\end{aligned}
$$

About these equations Dr. Chree remarks : "It is scarcely likely that these equations admit of a complete solution." * But the solution of them is of essential importance, for on it depends the determination of the exact change in the form of the cross section. I shall presently show that they can be solved. The terms in $\alpha_{3}, \beta_{3}$ will involve $\frac{a^{3}}{c^{3}}$, and thus will be relatively unimportant, so we shall not determine them here.

Let

$$
\left.\begin{array}{l}
u=\alpha_{2}^{\prime}+i \beta_{2}^{\prime}, \text { where } i=\sqrt{-1} \\
v=\alpha_{2}^{\prime}-i \beta_{2}^{\prime},
\end{array}\right\} \text {. }
$$

Multiply the equation (22) by $i$ : the two equations (21), (22) become, by addition and subtraction,

$$
\left.\begin{array}{l}
\frac{d u}{d t}-i \frac{\kappa^{\prime}}{2 \pi b^{2}} u=-i \frac{\kappa b}{2 \pi c^{2}} e^{2 i \epsilon}-\frac{b}{2 \pi c^{2}} \frac{d \sigma}{d t} e^{2 i \epsilon}, \\
\frac{d v}{d t}+i \frac{\kappa^{\prime}}{2 \pi b^{2}} v=i \frac{\kappa b}{2 \pi c^{2}} e^{-2 i \epsilon}-\frac{b}{2 \pi c^{2}} \frac{d \sigma}{d t} e^{-2 i \epsilon} .
\end{array}\right\} .
$$

These are linear differential equations. On integration we get

$$
\left.\begin{array}{rl}
u e^{-i \kappa^{\prime}} \int_{2 \pi b^{4}}^{d} & =u_{0}-\int_{0}^{t} e^{i\left(2 e-\frac{\kappa^{\prime}}{2 \pi} \int \frac{d t}{b^{2}}\right)}\left\{i \frac{\kappa b}{2 \pi c^{2}}+\frac{b}{2 \pi c^{2}} \frac{d \sigma}{d t}\right\} d t \\
v e^{i \frac{\kappa^{\prime}}{2 \pi} \int_{b 2}^{d t}}=v_{0}+\int_{0}^{t} e^{-i\left(2 \epsilon-\frac{x^{\prime}}{2 \pi} \int \frac{d b}{b 2}\right)}\left\{i \frac{\kappa b}{2 \pi c^{2}}-\frac{b}{2 \pi c^{2}} \frac{d \sigma}{d t}\right\} d t
\end{array}\right\}
$$

where $u_{0}$ and $v_{0}$ are initial values of $u$ and $v$. If ${ }_{0} \alpha_{2}{ }^{\prime},{ }_{0} \beta_{2}{ }^{\prime}$ be the values of $\alpha_{2}^{\prime}, \beta_{2}^{\prime}$ respectively when $t=0$, we must have

$$
u_{0}={ }_{0} \alpha_{2}{ }^{\prime}+i_{0} \beta_{2}{ }^{\prime}, \quad v_{0}={ }_{0} \alpha_{2}{ }^{\prime}-i_{0} \beta_{2}{ }^{\prime} . . \quad \text {. }
$$

* L. c. pp. 115, 118. 
Compressible Fluid moving in Incompressible Liquid. 145

Now, substituting the values of $u$ and $v$ from (23) in (25), we get by addition and subtraction,

$$
\begin{aligned}
& \alpha_{2}^{\prime} \cos \left(\frac{\kappa^{\prime}}{2 \pi} \int \frac{d t}{b^{2}}\right)+ \beta_{2}{ }^{\prime} \sin \left(\frac{\kappa^{\prime}}{2 \pi} \int \frac{d t}{b^{2}}\right) \\
&={ }_{0} \boldsymbol{\alpha}_{2}{ }^{\prime}+\int_{0}^{t}\left[\frac{\kappa b}{2 \pi c^{2}} \sin \left(2 \epsilon-\frac{\kappa^{\prime}}{2 \pi} \int \frac{d t}{b^{2}}\right)\right. \\
&\left.-\frac{b}{2 \pi c^{2}} \frac{d \sigma}{d t} \cos \left(2 \epsilon-\frac{\kappa^{\prime}}{2 \pi} \int \frac{d t}{b^{2}}\right)\right] d t \\
& \alpha_{2}{ }^{\prime} \sin \left(\frac{\kappa^{\prime}}{2 \pi} \int \frac{d t}{b^{2}}\right)-\beta_{2}{ }^{\prime} \cos \left(\frac{\kappa^{\prime}}{2 \pi} \int \frac{d t}{b^{2}}\right) \\
&=-{ }_{0} \beta_{2}{ }^{\prime}+\int_{0}^{t}\left[\frac{\kappa b}{2 \pi c^{2}} \cos \left(2 \epsilon-\frac{\kappa^{\prime}}{2 \pi} \int \frac{d t}{b^{2}}\right)\right. \\
&\left.\quad+\frac{b}{2 \pi c^{2}} \frac{d \sigma}{d t} \sin \left(2 \epsilon-\frac{\kappa^{\prime}}{2 \pi} \int \frac{d t}{b^{2}}\right)\right] d t .
\end{aligned}
$$

Solving these equations for $\alpha_{2}{ }^{\prime}$ and $\beta_{2}{ }^{\prime}$, we get

$$
\begin{gathered}
\alpha_{2}{ }^{\prime}={ }_{0} \alpha_{2}^{\prime} \cos \left(\frac{\kappa^{\prime}}{2 \pi} \int \frac{d t}{b^{2}}\right)-{ }_{0} \beta_{2}{ }^{\prime} \sin \left(\frac{\kappa^{\prime}}{2 \pi} \int \frac{d t}{b^{2}}\right) \\
+\cos \left(\frac{\kappa^{\prime}}{2 \pi} \int \frac{d t}{b^{2}}\right) \int_{0}^{t} \frac{b}{2 \pi c^{2}}\left[\kappa \sin \left(2 \epsilon-\frac{\kappa^{\prime}}{2 \pi} \int \frac{d t}{b^{2}}\right)\right. \\
\left.\quad-\frac{d \sigma}{d t} \cos \left(2 \epsilon-\frac{\kappa^{\prime}}{2 \pi} \int \frac{d t}{b^{2}}\right)\right] d t \\
+\sin \left(\frac{\kappa^{\prime}}{2 \pi} \int \frac{d t}{b^{2}}\right) \int_{0}^{t} \frac{b}{2 \pi c^{2}}\left[\kappa \cos \left(2 \epsilon-\frac{\kappa^{\prime}}{2 \pi} \int \frac{d t}{b^{2}}\right)\right. \\
\left.+\frac{d \sigma}{d t} \sin \left(2 \epsilon-\frac{\kappa^{\prime}}{2 \pi} \int \frac{d t}{b^{2}}\right)\right] d t, \\
\beta_{2}^{\prime}={ }_{0} \alpha_{2}{ }^{\prime} \sin \left(\frac{\kappa^{\prime}}{2 \pi} \int \frac{d t}{b^{2}}\right)+{ }_{0} \beta_{2}{ }^{\prime} \cos \left(\frac{\kappa^{\prime}}{2 \pi} \int \frac{d t}{b^{2}}\right) \\
+\sin \left(\frac{\kappa^{\prime}}{2 \pi} \int \frac{d t}{b^{2}}\right) \int_{0}^{t} \frac{b}{2 \pi c^{2}}\left[\kappa \sin \left(2 \epsilon-\frac{\kappa^{\prime}}{2 \pi} \int \frac{d t}{b^{2}}\right)\right. \\
\left.-\frac{d \sigma}{d t} \cos \left(2 \epsilon-\frac{\kappa^{\prime}}{2 \pi} \int \frac{d t}{b^{2}}\right)\right] d t \\
-\cos \left(\frac{\kappa^{\prime}}{2 \pi} \int \frac{d t}{b^{2}}\right) \int_{0}^{t} \frac{b}{2 \pi c^{2}}\left[\kappa \cos \left(2 \epsilon-\frac{\kappa^{\prime}}{2 \pi} \int \frac{d t}{b^{2}}\right)\right] \\
\left.+\frac{d \sigma}{d t} \sin \left(2 \epsilon-\frac{\kappa^{\prime}}{2 \pi} \int \frac{d t}{b^{2}}\right)\right] d t .
\end{gathered}
$$

Phil. Mag. Ser. 6. Vol, 40. No. 235. July 1920. 
146 Mr. B. Datta on Stability of two Rectilinear Vortices of

In our further investigations, we should remember that if $\epsilon=0$ when $t=0$, it can be proved that *

$$
\epsilon=\frac{\kappa+\kappa^{\prime}}{2 \pi} \int_{e_{0}}^{t} \frac{d t}{c_{0}{ }^{2}+\left(\sigma+\sigma^{\prime}-\sigma_{0}-\sigma_{0}{ }^{\prime}\right) / \pi}, .
$$

where $c_{0}, \sigma_{0}, \sigma_{0}^{\prime}$ are the values of $c, \sigma, \sigma^{\prime}$ respectively when $t=0$.

The equations (27) and (28) show that there are two distinet types of vibrations of $\alpha_{2}^{\prime}$ and $\beta_{2}{ }^{\prime}$ of gradually virying periods given by

$$
\frac{\kappa+\kappa^{\prime}}{\pi} \int_{0}^{t} \frac{\frac{\kappa^{\prime}}{2 \pi \pi_{0}} \int_{0}^{t} \frac{d t}{b^{2}}=2 n \pi, \quad . \quad . . .}{\frac{d t}{c_{0}{ }^{2}+\left(\sigma+\sigma^{\prime}-\sigma_{0}-\sigma_{0}{ }^{\prime}\right) / \pi}}=2 n \pi, \quad .
$$

where $n$ is any integer. Evidently the period given by (30) is shorter than the period given by (31). Suppose ${ }_{0} \alpha_{2}{ }^{\prime}={ }_{0} \beta_{2}{ }^{\prime}=0 ;$ since $2 \epsilon-\frac{\kappa^{\prime}}{2 \pi} \int \frac{d t}{b^{2}}$ is always
negative, we get

$$
\begin{aligned}
\alpha_{2}^{\prime}= & -\cos \left(\frac{\kappa^{\prime}}{2 \pi} \int \frac{d t}{b^{2}}\right) \int_{0}^{t} \frac{b}{2 \pi c^{2}}\left[\kappa \sin \left(\frac{\kappa^{\prime}}{2 \pi} \int_{b^{2}}^{d t}-2 \epsilon\right)\right. \\
& +\frac{d \sigma}{d t} \cos \left(\frac{\kappa^{\prime}}{2 \pi}\left(\frac{d t}{b^{2}}-2 \epsilon\right)\right] d t \\
& +\sin \left(\frac{\kappa^{\prime}}{2 \pi} \int \frac{d t}{l^{2}}\right) \int_{0}^{t} \frac{b}{2 \pi \epsilon^{2}}\left[\kappa \operatorname { c o s } \left(\frac{\kappa^{\prime}}{2 \pi}\left(\frac{d t}{b^{2}}-2 \epsilon\right)\right.\right. \\
& -\frac{d \sigma}{d t} \sin \left(\frac{\kappa^{\prime}}{2 \pi}\left(\frac{d t}{b^{2}}-2 \epsilon\right)\right] d t,(32)
\end{aligned}
$$

so that vibrations are becoming more and more prominent as the rortices are approaching one another; since

$$
\left(\frac{\kappa^{\prime}}{2 \pi} \int \frac{d t}{b^{2}}-2 \epsilon\right)
$$

is always real and $b / c$ is also small, the value of $\alpha_{2}^{\prime}$ cannot increase indefinitely so that the motion is stable $\nmid$.

We shall now proceed to consider some interesting particular cases.

* Chree, $l$. e. p. 109.

$\dagger$ This point will be clearer from equations (25) which are of the type

$$
u e^{i f_{1}(t)}=u_{0}-\int_{0}^{t} e^{i f_{2}(t)} \mathrm{F}\left(\frac{b}{a^{2}}\right) d t .
$$

Since $\frac{d c^{2}}{d t}=2 \frac{d}{d t} b^{2}$ (Chree, p. 108), the function $\mathrm{F}\left(\frac{b}{c^{2}}\right)$ continually decreases as $t$ increases so that in the limit the integral must be finite. 
Compressible Fluid moving in Incompressible Liquid. 147

Case I.-Two columns of fuids of rarying density possessing no vorticity.

In this case

$$
\kappa=\kappa^{\prime}=\epsilon=0,
$$

so that the equations (27) and (28) become

$$
\begin{aligned}
& \alpha_{2}{ }^{\prime}={ }_{0} \alpha_{2}{ }^{\prime}-\int_{0}^{t} \frac{b}{2 \pi c^{2}} \frac{d \sigma}{d t} d t, \\
& \beta_{2}{ }^{\prime}={ }_{0} \beta_{2}{ }^{\prime} ;
\end{aligned}
$$

which agree with the result obtained by Dr. Chree. Since $\frac{d \sigma}{d t}=2 \pi a \frac{d a}{d t}, \alpha_{2}^{\prime}$ would increase or decrease according as $a$ was decreasing or increasing. "Thus, if both columns were diminishing in cross section, and so approaching, there would be a decided tendency in both cross sections to assume an elliptical sort of outline, the major axes coinciding with the line joining their centres."

\section{Case 11 .- Two rectilinear vortices in an incompressible fluid.}

As previously mentioned, this case has been studied by Sir J. J. Thomson. We shall simply show that his results can be deduced from our more general results. Let ${ }_{0} \alpha_{2}{ }^{\prime}={ }_{0} \beta_{2}{ }^{\prime}=0$; also if $n$ be the angular velocity of the central line, $\epsilon=n t$. Then putting $\frac{d \sigma}{d t}=0$ in (27) we get, since $\kappa^{\prime}=\zeta^{\prime} \pi l^{2}, \kappa=\zeta \pi u^{2}$,

$$
\begin{aligned}
\alpha_{2}{ }^{\prime}=\cos \left(\frac{\zeta^{\prime}}{2} t\right) \int_{0}^{t} \frac{\zeta a^{2} b}{2 c^{2}} \cdot \sin \left(2 n t-\frac{\zeta^{\prime}}{2} t\right) d t \\
\quad+\sin \left(\frac{\zeta^{\prime}}{2} t\right) \int_{0}^{t} \frac{\zeta a^{2} b}{2 c^{2}} \cos \left(2 n-\frac{\zeta^{\prime}}{2}\right) t d t
\end{aligned}
$$

or $\quad \alpha_{2}^{\prime}=\frac{\zeta^{\prime} a^{2} b}{2 c^{2}\left(\frac{\zeta^{\prime}}{2}-2 n\right)}\left\{\cos 2 n t-\cos \left(\frac{\zeta^{\prime}}{2} t\right)\right\}$;

similarly from (28),

$$
\beta_{2}{ }^{\prime}=\frac{\zeta^{\prime} a^{2} b}{2 c^{2}\left(\frac{\zeta^{\prime}}{2}-2 n\right)}\left\{\sin 2 n t-\sin \left(\frac{\zeta^{\prime}}{2} t\right)\right\} .
$$

These will be found identical with the results obtained by Sir J. J. I'homson * when we remember that $\zeta=2 \omega$, and $\zeta^{\prime}=2 \omega^{\prime}$.

* 'Motion of Vortex Rings, p. 77. 
148 Stability of Rectilinear Vortices of Compressible Fluid.

Case III.-A Vortex-pair.

$A$ combination of two equal and opposite vortices is called a "vortex-pair." Put $\kappa=-\kappa^{\prime}, a=b$; then $\epsilon=0$ : we have from $(32)$

$$
\begin{aligned}
\alpha_{2}^{\prime}=\cos \left(\frac{\kappa^{\prime}}{2 \pi} \int \frac{d t}{b^{2}}\right) \int_{0}^{t} \frac{b}{2 \pi c^{2}} & {\left[\kappa^{\prime} \sin \left(\frac{\kappa^{\prime}}{2 \pi} \int \frac{d t}{b^{2}}\right)\right.} \\
& \left.-\frac{d \sigma^{\prime}}{d t} \cos \left(\frac{\kappa^{\prime}}{2 \pi} \int \frac{d t}{b^{2}}\right)\right] d t \\
-\sin \left(\frac{\kappa^{\prime}}{2 \pi} \int \frac{d t}{b^{2}}\right) \int_{0}^{t} b \quad 2 \pi c^{2} & {\left[\kappa^{\prime} \cos \left(\frac{\kappa^{\prime}}{2 \pi} \int \frac{d t}{b^{2}}\right)\right.} \\
+ & \left.\frac{d \sigma^{\prime}}{d t} \sin \left(\frac{\kappa^{\prime}}{2 \pi} \int \frac{d t}{b^{2}}\right)\right] d t ;
\end{aligned}
$$

so that in this case, the vortices have a single type of disturbance of period given by

$$
\frac{\kappa^{\prime}}{2 \pi} \int \frac{d t}{b^{2}}=2 \pi n
$$

As a vortex-pair is the two-dimensional analogue of a circular vortex-ring some of the properties of the latter may be deduced from it.

\section{Case $Y$ r.-A single vorter parallel to an infinite wall.}

This is really a subcase of Case III. For the velocity of the fluid at all points of the plane of symmetry is wholly tangential, so that this plane may be supposed to form a rigid boundary of the fluid on either side of it; thus we obtain the case of a single rectilinear vortex in the neighbourhood of a fixed plane wall to which it is parallel. If $h$ be the distance of the vortex from the wall we get

$$
\begin{aligned}
& \alpha_{2}^{\prime}=\cos \left(\frac{\kappa^{\prime}}{2 \pi} \int \frac{d t}{b^{2}}\right) \int_{0}^{t} \frac{b}{8 \pi h^{2}} {\left[\kappa^{\prime} \sin \left(\frac{\kappa^{\prime}}{2 \pi} \int \frac{d t}{b^{2}}\right)\right.} \\
&\left.-\frac{d \sigma^{\prime}}{d t} \cos \left(\frac{\kappa^{\prime}}{2 \pi} \int \frac{d t}{b^{2}}\right)\right] d t \\
&-\sin \left(\frac{\kappa^{\prime}}{2 \pi} \int \frac{d t}{b^{2}}\right) \int_{0}^{t} \frac{b}{8 \pi h^{2}}\left[\kappa^{\prime} \cos \left(\frac{\kappa^{\prime}}{2 \pi} \int \frac{d t}{b^{2}}\right)\right. \\
&\left.+\frac{d \sigma^{\prime}}{d t} \sin \left(\frac{\kappa^{\prime}}{2 \pi} \int \frac{d t}{b^{2}}\right)\right] d t .
\end{aligned}
$$

Hence the diameter parallel to the wall is greater if $b$ is increasing, $i . e$. the vortex is receding from the wall and vice versa.

University of Calcutta, Ist September, 1919. 